\title{
Educação permanente em saúde: Estratégia de prevenção e controle de infecção hospitalar
}

RESUMO | Objetivo: analisar através da literatura as estratégias da educação permanente que contribuem para a mudança de condutas de prevenção e controle de infecção em assistência à saúde pela equipe multidisciplinar. Método: Revisão integrativa com busca nas bases de dados LILACS, MEDLINE e BDENF. Elaborou-se um instrumento de coleta de dados que sinaliza as características para análise dos estudos. Resultados: Incluíram-se 12 artigos e foram agrupados por similaridade didática de acordo com as estratégias implementadas quanto à prevenção e controle em assistência à saúde. Conclusão: Ficou evidente que a utilização de diversas estratégias da educação permanente, na assistência à saúde, traz melhorias à medida que estimulam os profissionais no aperfeiçoamento, qualificação e na atualização de conceitos e ações práticas, contribuindo também para o desenvolvimento da reflexão crítica, trazendo mudança comportamental, favorecendo desta forma o crescimento pessoal e profissional e a interação da equipe multidisciplinar, propiciando assim a melhora na qualidade da assistência.

Palavras-chaves: Pessoal de Saúde; Educação Continuada/Permanente; Controle de Infecções.

ABSTRACT | Objective: analyze through the literature the strategies of the permanent education that contribute in the change of conduits of prevention and control of infection in health care by the multidisciplinary team. Method: Integrative revision with search in LILACS, MEDLINE and BDENF databases. Elaborated an instrument of data collection that signals the characteristics for analysis of the studies. Results: Were included 12 articles and grouped by didactic similarity according to the strategies implemented regarding prevention and control in health care. Conclusion: It was evident that the use of several strategies of permanent education in health care, brings improvements as they stimulate professionals in the improvement, qualification and updating of concepts and practical actions, also contributing to the development of critical reflection, bringing change behavioral, thus favoring personal and professional growth and the interaction of the multidisciplinary team, thus improving the quality of care.

Keywords: Health Personnel; Continuing/ Permanent Education; Infection Control.

RESUMEN | Objetivo: analizar a través de la literatura las estrategias de la educación permanente que contribuyen en el cambio de conductas de prevención y control de infección en asistencia a la salud por el equipo multidisciplinario. Método: Revisión integrativa con búsqueda en las bases de datos LILACS, MEDLINE y BDENF. Elaboró un instrumento de recolección de datos que señaliza las características para el análisis de los estudios. Resultados: Se incluyeron 12 artículos y fueron agrupados por similitud didáctica de acuerdo con las estrategias implementadas en cuanto a la prevención y control en asistencia a la salud. Conclusión: Es evidente que la utilización de diversas estrategias de la educación permanente en la asistencia a la salud, trae mejoras a medida que estimulan a los profesionales en el perfeccionamiento, calificación y actualización de conceptos y acciones prácticas, contribuyendo también al desarrollo de la reflexión crítica, trayendo cambio comportamental, favoreciendo de esta forma el crecimiento personal y profesional y la interacción del equipo multidisciplinario propiciando así la mejora en la calidad de la asistencia

Descriptores: Personal de Salud; Educación Continua/ Permanente; Control de Infecciones.

Mônica Aparecida de Oliveira Pinto Porto

Enfermeira Especializanda em Controle de Infecção em Assistência à Saúde da Escola de Enfermagem Aurora de Afonso Costa. Universidade Federal Fluminense

\section{Maritza Consuelo Ortiz Sanchez}

Enfermeira. Dra. em Enfermagem. Professora Adjunto do Departamento de Fundamento de Enfermagem e Administração da Escola de Enfermagem Aurora de Afonso Costa Universidade Federal Fluminense.

\section{Maria Lelita Xavier}

Doutora em Enfermagem. Professora da Faculdade de Enfermagem, da Universidade do Estado do Rio de Janeiro, Rio de Janeiro - RJ, Brasil.

Recebido em: 30/05/2019

Aprovado em: 30/05/2019

\section{Miriam Marinho Chrizostimo}

Enfermeira. Doutora em Enfermagem. Departamento de Fundamentos de Enfermagem e Administração. Universidade Federal Fluminense (UFF), Escola de Enfermagem Aurora de Afonso Costa (EEAAC). Niterói-RJ, Brasil.

\section{Euzeli da Silva Brandão}

Enfermeira, Professora Doutora no Programa de Pós-Graduaçāo,Universidade Federal Fluminense

\section{Márcia Valéria Rosa Lima}

Enfermeira. Doutora em Enfermagem. Departamento de Enfermagem MédicoCirúrgico (MEM) Universidade Federal Fluminense (UFF), Escola de Enfermagem Aurora de Afonso Costa (EEAAC). NiteróiRJ, Brasil.
INTRODUÇÃO

$\Lambda$ s ações de controle e prevenção de infecção relacionadas à assistência à saúde vêm sendo empregadas há centenas de anos, entretanto, a alta incidência de casos clínicos relacionados à infecção em assistência à saúde ainda continua sendo destacada, tendo sido reportada pela Organização Mundial da Saúde (OMS) que, 1 em 10 pacientes adquirem uma infecção durante a assistência prestada ${ }^{(1-4)}$, permanecendo, assim, a alta incidência um problema de grande relevância nos dias atuais.

Ainda que as estimativas mundiais da infecção em assistência à saúde não este- 
jam disponíveis, de acordo com os dados dos estudos publicados, ficam nítidas as evidências de que centenas de milhões de pacientes no mundo são afetados a cada ano, sendo que a prevalência da doença é maior em populações de baixa e média rendas ${ }^{(5)}$.

Esse fato se confirma no relatório do Banco Mundial - Organização e Desenvolvimento Econômico (OCDE) e da OMS, que traz uma visão geral da assistência à saúde mundialmente, em que se evidencia que $10 \%$ dos pacientes adquirirá infecção hospitalar durante a internação em países de baixa renda e $7 \%$ dos pacientes em países de renda alta. Podendo ser em decorrência de erros de medicação, tratamento desnecessário, assistência clínica inadequada e ausência de treinamento da equipe de saúde, sendo uma realidade mundial tanto para baixa renda quanto para alta renda ${ }^{(6)}$.

Há forte incidência de que as infecções podem ser evitadas e o ônus reduzido em até $50 \%$ ou mais. As infecções em assistência à saúde devem ser tratadas de forma prioritária e por meio de amplas abordagens eficientes visando a segurança do paciente. O programa de segurança do paciente, em conjunto com outros Programa da OMS, auxiliam, avaliam, planejam e implementam as políticas de controle e prevenção da infecção e incluem ações oportunas em nível nacional e em níveis institucionais para reduzir as infecções ${ }^{(5)}$.

$\mathrm{Na}$ década de 1960, surgiram as primeiras Comissões de Controle de Infecção Hospitalar (CCIH) no Brasil e, gradativamente, por meio de ações e trabalho contínuo dos profissionais envolvidos, obteve-se nova concepção: Infecções Relacionadas à Assistência à Saúde (IRAS). Concluiu-se que essas infecções não se dariam somente pela assistência hospitalar, mas também tendo riscos de adquiri-las em procedimentos ambulatoriais, nas instituições de longa permanência, instituições para doentes crônicos, "home care", serviços de hemodiálise e clínicas odontológicas ${ }^{(7)}$.

Em 2004, o Ministério da Saúde assumiu a responsabilidade com a formação e capacitação dos trabalhadores de saúde, reconhecendo por meio da Portaria GM/MS n. ${ }^{\circ}$ 198/2004 a Política Nacional de Educação Permanente em Saúde (PNEPS), cuja qual foi instituída com a intenção de gerar condições favoráveis para a formação e desenvolvimento dos recursos humanos para a saúde ${ }^{(8)}$.

Em 2006, o Governo Federal instituiu o Pacto pela Saúde da Portaria GM/MS n. ${ }^{\circ}$ 399/06, o qual apresenta modificações na gestão da Educação Permanente em Saúde (EPS), sendo esta reestruturada em 2007 com a instituição da Portaria n. ${ }^{\circ}$ 1996/07/GM/MS ${ }^{(1,2)}$, cuja proposta é qualificar e transformar as práticas de saúde e, com isso, prestar cuidado integral ao ser humano. As ações da EPS têm intuito de efetivar as práticas reflexivas, éticas, críticas e humanísticas visando à equipe e ao ambiente do trabalho ${ }^{(9)}$.

\section{Tabela 1. Descrição da estratégia PICo. Niterói, RJ, Brasil, 2019}

\begin{tabular}{ll} 
Acrônimo & Descrição \\
P & Pessoal de Saúde \\
I & Educação Continuada \\
C & Controle de Infecções \\
\hline 0 & Melhoria na assistência e redução do nível de infecção hospitalar
\end{tabular}

A Educação Permanente é a atualização das práticas do dia a dia, favorecendo o crescimento das relações e processos que surgem do interior das equipes ${ }^{(9)}$.

Esse estudo tem como objetivo analisar através da literatura as estratégias da educação permanente que contribuem para a mudança de condutas de prevenção e controle de infecção em assistência à saúde pela equipe multidisciplinar.

\section{METODOLOGIA}

Essa pesquisa utiliza o método de revisão integrativa, sendo assim realizada por meio das seguintes etapas: elaboração da questão de pesquisa, busca na literatura, coleta de dados, análise críti- ca dos artigos, discussão dos resultados, apresentação da revisão e conclusão.

Elaborou-se na primeira etapa, a questão norteadora com a seguinte indagação: Quais as estratégias utilizadas pela educação permanente contribuem para a mudança de condutas da equipe multidisciplinar para prevenção e controle de infecção em assistência à saúde?

A estratégia de busca foi realizada através do portal da Biblioteca Virtual em Saúde (BVS). Para iniciação do levantamento nessa base, foi aplicada a estratégia PICo.

Foram utilizados para a seleção das produções os seguintes descritores: "Pessoal de saúde" ("Health Personnel") "AND" "Educação Continuada" ("Continuing Education") "OR" "Educação Permanente" "AND" "Controle de Infecções" ("Infection Control"). Utilizou-se a terminologia "Educação continuada" devido ao termo "Educação Permanente" não ser reconhecida pelos Descritores em Ciências da Saúde (DeCS). 
Critérios de exclusão: estudos duplicados e incompletos que não atendem à questão do estudo.

Elaboraram-se instrumentos que compreendem os dados de identificação dos artigos. Os artigos extraídos estão em ordem de acordo com a letra inicial das bases de dados utilizadas, seguidos do número conforme descrito nas referências bibliográficas. Realizou-se também o agrupamento dos artigos por características de semelhanças didáticas referentes à contribuição da educação permanente, que promovem prevenção e controle das IRAs. Com ênfase no delineamento dos estudos e com base nas características das pesquisas, os artigos foram hierar- quizados de acordo com cinco níveis de evidências descritas na tabela de Oxford Centre for Evidence-Based Medicine ${ }^{(10)}$.

\section{RESULTADOS}

Esse estudo possibilitou a análise de 12 artigos na íntegra, que atenderam aos critérios de inclusão previamente estabelecidos. A busca pelas produções científicas se deu até o dia 26 de fevereiro de 2019 e resultou num total de 7357 artigos publicados. Destes, 7192 contidos na base de dados da MEDLINE, 128 na LILACS e 37 na BDENF. Dentre estes, foram excluídas 5147 publicações por não apresentarem textos completos e 182
Figura 1. Fluxograma da estratégia de busca e seleção dos artigos nas bases de dados da Biblioteca Virtual em Saúde (BVS). Niterói, RJ, Brasil, 2019

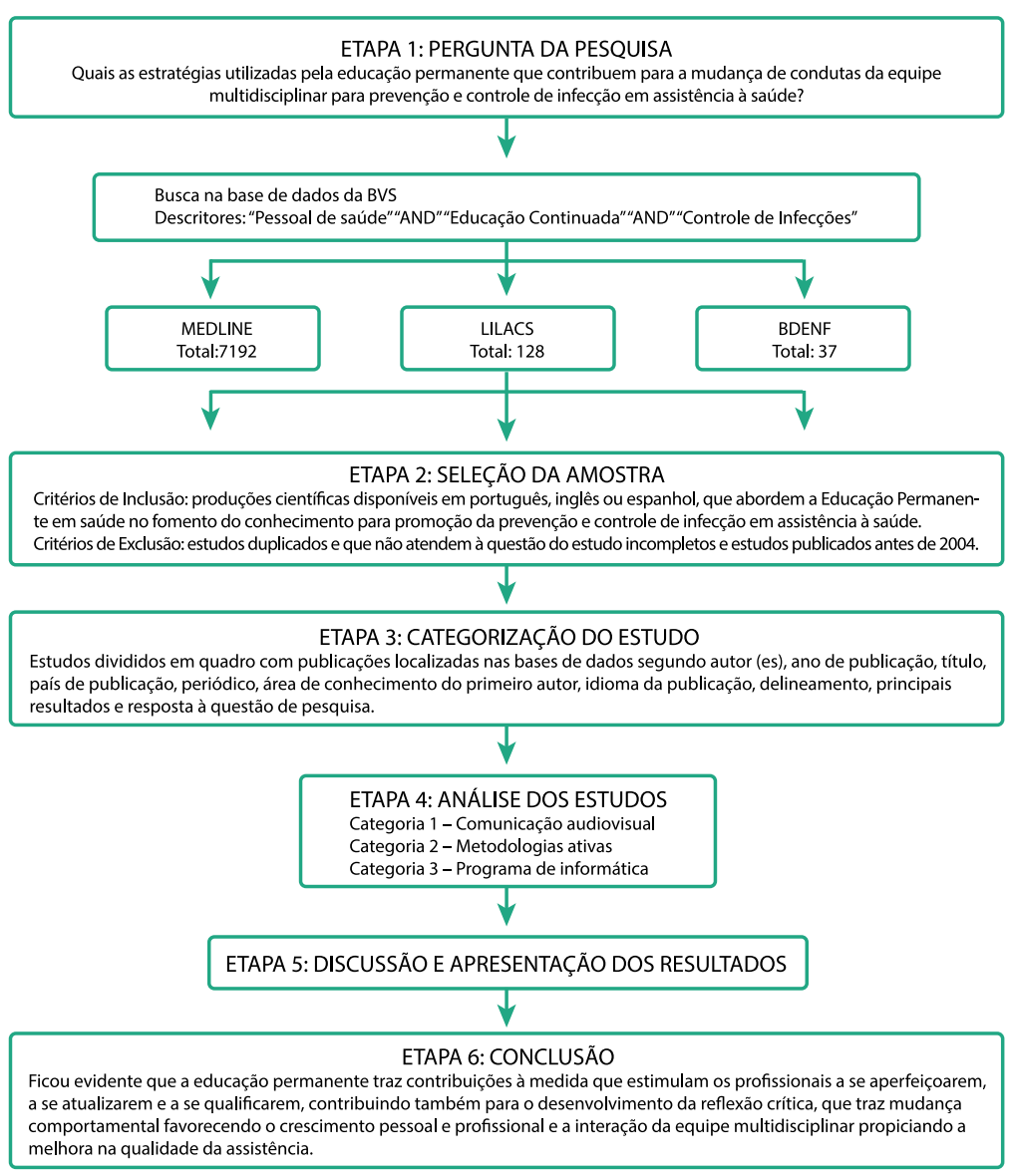

publicações foram excluídas por idioma. Após essas exclusões, foi realizada a leitura dos títulos e resumos das publicações com o propósito de analisar a relevância do estudo para sua inclusão na amostra. Em seguida, excluíram-se 15 artigos por motivo de duplicação e 2001 artigos por não responderem à questão da pesquisa. Dessa forma, 12 artigos foram incluídos, sendo 08 artigos da base de dados da MEDLAINE, 02 artigos da base LILACS e 02 artigos da base BDENF para a construção dessa revisão integrativa.

Em relação às informações sobre a composição dos estudos das amostras analisadas, evidencia-se que $83,34 \%$ dos artigos eram artigos originais, enquanto $16,66 \%$ dos artigos eram relatos de experiência.

Quanto ao idioma de origem no qual o artigo foi publicado, observa-se um predomínio do idioma português em $66,66 \%$ dos artigos, do idioma inglês em $33,34 \%$ e do idioma espanhol não foi encontrado nenhum artigo.

Quanto ao ano de publicação, os artigos selecionados foram entre os anos 2004 a 2018 e verificou-se um maior volume de pesquisas nos últimos dez anos, sendo 02 artigos no mesmo ano citados a seguir: 2009 (16,66\%), 2010 $(16,66 \%)$ e $2017(16,66 \%)$. Nos seguintes anos obteve-se 01 artigo a cada ano respectivamente: 2004 (8,33\%), 2006 $(8,33 \%), 2007$ (8,33\%), 2011 (8,33\%), $2013(8,33 \%)$ e $2015(8,33 \%)$. Entre os anos de 2005, 2008, 2012, 2014, 2016 e 2018 não se obteve estudo que respondesse à questão da pesquisa.

Ao que se refere ao periódico de publicação, 66,66\% dos artigos estão publicados nas revistas dirigidas ao campo da enfermagem, 16,66\% estão voltadas na área da infectologia e 8,33\% dos artigos estão publicados nas revistas das áreas da medicina e microbiologia, respectivamente em cada um. Ao que concerne ao país de origem dos estudos, 66,66\% dos artigos são oriundos da América do Sul; enquanto 8,33\% são respectivamente da América do Norte, Continente Asiático e Oceania. 
Considerando-se a formação profissional dos autores dos estudos, 50\% dos artigos foram redigidos exclusivamente por enfermeiros, 25\% exclusivamente redigidos por médicos, 8,33\% foram redigidos por um enfermeiro e um médico, 8,33\% redigidos por um médico e um microbiologista e $8,33 \%$ redigido por infectologistas. Verificou-se que a área de enfermagem foi a que mais produziu e publicou sobre o tema educação permanente.

Quanto à similaridade didática das metodologias educativas empregadas, $58,33 \%$ dos artigos analisados utilizaram cartazes e pôsteres que promovem mudanças de condutas nos processos de educação permanente em saúde para o controle das infecções hospitalares.

Quanto à qualidade da evidência, observou-se a predominância dos artigos classificados como: NE $1 \mathrm{~A}$ com $50 \%$ dos artigos; NE 2A 8,3\% artigos; NE 2C com $25 \%$ dos artigos e NE 4 com $16,3 \%$ artigos. Dessa forma, percebe-se que $83,3 \%$ dos artigos estão caracterizados entre NE de $1 \mathrm{~A}$ e $2 \mathrm{C}$ possuindo delineamento adequado que nos mostram confiabilidade e precisão em condutas para a prevenção e controle da infecção em assistência à saúde.

O esquema de categorização dos artigos e a classificação dos níveis de evidências são descritos na Figura 2, relacionando a especificação de cada categoria com o grupo de artigos que nelas se enquadram e seus respectivos níveis de evidências classificados.

\section{Figura 2. Categorização dos artigos e a classificação dos níveis de evidências. Niterói, RJ, Brasil, 2019}

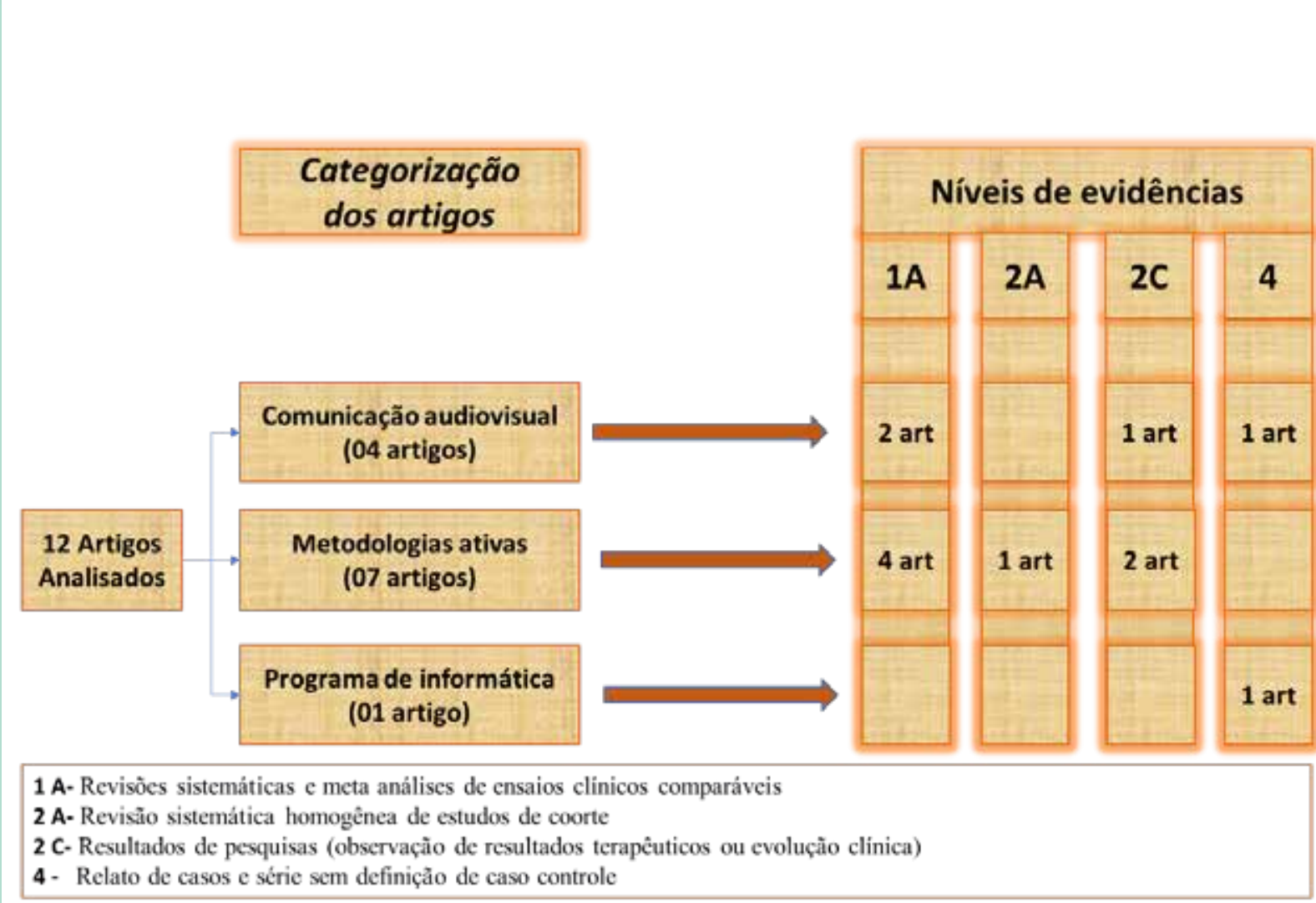


Os Quadros abaixo 1 e 2 sumarizam as principais características dos estudos revisados no que tange à educação permanente para promoção da prevenção e controle de infecção na assistência à saúde com finalidade de categorizar esses artigos contendo os seguintes aspectos: identificação dos artigos/autor/título/objetivo/ resposta à questão da pesquisa.

\section{DISCUSSÃO}

Ao se defrontar cada vez mais com as consequências decorrentes da infecção hospitalar $(\mathrm{IH})$, torna-se necessário elaborar ações que visem o controle e prevenção. Dessa forma, as pesquisas evidenciam que a aplicação de estratégias que levem o profissional a atuar de forma crítica e reflexiva, sendo fundamental para obter efeitos positivos que os estimulem a repensar as práticas cotidianas, favorecendo a transformação das atividades e contribuindo na qualidade dos serviços de saúde.

Dentre os $66 \%$ de artigos de origem brasileira, observou-se que apenas um estudo mencionou a PNEPS. Apesar dos demais não citarem a PNEPS, utilizaram ações no intuito de fundir as práticas de forma reflexiva e crítica visando a equipe e o ambiente do trabalho, em concor-

Quadro 1. Descrição e análise dos estudos. Niterói, RJ, Brasil, 2019

Ord Autor/Ano $\quad$ Título $\quad$ Resposta a questão de pesquisa

$M^{17} \quad$ Musu, $M$, et Controlling cateter-related blood stream infections trough a al. (2017) multi-centre education programe for intensive care units

\begin{tabular}{|c|c|c|}
\hline$M^{12}$ & $\begin{array}{l}\text { Kapil R.et } \\
\text { al. (2015) }\end{array}$ & $\begin{array}{l}\text { Hand hygiene in reducing transient flora on the hands of } \\
\text { health care workers: An education intervention }\end{array}$ \\
\hline$M^{13}$ & $\begin{array}{l}\text { Ross,A.et al. } \\
2006 .\end{array}$ & $\begin{array}{l}\text { The impacto f na evidence-based practice education on the role } \\
\text { of oal care in the prevention of ventilator-associated pneumonia }\end{array}$ \\
\hline
\end{tabular}

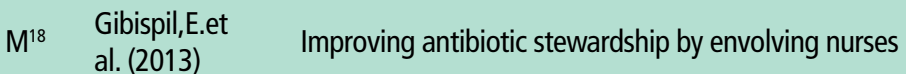

$\begin{array}{cc}\text { M } 14 & \begin{array}{l}\text { Zilah,N.et } \\ \text { al. (2006) }\end{array} \\ & \text { incentivo à adesão entre os profissionais de saúde de uma } \\ \text { Unidade de Terapia Intensiva Neonatal. }\end{array}$

M ${ }^{19}$ Valente $\mathrm{G}$. $\quad 0$ enfermeiro na educação em saúde do trabalhador da et al (2011) limpeza no ambiente hospitalar
Exposição de materiais (pôsteres e fichas informativas), treinamento e desempenho contínuo e programa de vigilância.

Exposição de cartazes da OMS; Ensino interativo na prática e aplicação de questionário para avaliação sobre a percepção da Higiene das mãos

Cartazes, Story boards e ênfase no Protocolo de cuidados bucais.

Capacitação dos enfermeiros na gestão de antibióticos; Reunião com apresentação de slides e discussão; Fornecimento de folhetos; Visitação diária à enfermaria pelo profissional do Centro de controle de infecção para esclarecimento de dúvidas.

Paródias musicais com a temática da Higienização das mãos; Cartazes informativos estilizados; Divulgação de dez frases elaboradas pela equipe de saúde através do serviço de radiofonia.

Aplicação de questionário com perguntas especificas acerca da limpeza hospitalar e fornecimento de folders após a análise das respostas com intuito de potencializar o conhecimento e orientar as atividades dos profissionais.

Programa educativo virtual com a intenção de o profissional levar à reflexão crítica de suas atividades diárias e permitindo a interação e expondo suas ideias e de forma conjunta propi-

ciar a reconstrução dos trabalhos.
M 24 Silva,LMG. Ambiente virtual de aprendizagem na educação continuada et al. (2009) em enfermagem

\begin{tabular}{|c|c|c|c|}
\hline$L^{15}$ & $\begin{array}{l}\text { Neves,ZCP. } \\
\text { et al. (2009) }\end{array}$ & $\begin{array}{l}\text { Relato de experiência: utilização de cartazes estilizados } \\
\text { como medida de incentivo à higienização das mãos. }\end{array}$ & Cartazes estilizados \\
\hline$M^{20}$ & $\begin{array}{l}\text { Souza,MCB. } \\
\text { et al. }(2004)\end{array}$ & $\begin{array}{l}\text { Enfermagem no centro de material esterilizado - a prática } \\
\qquad \text { da educação continuada }\end{array}$ & Realização de entrevista para cada membro da equipe de saúde. \\
\hline$B^{21}$ & $\begin{array}{l}\text { Jacondino,- } \\
\text { CB. et al. } \\
\text { (2010) }\end{array}$ & $\begin{array}{l}\text { Educação em serviço: Qualificação da equipe de enferma- } \\
\text { gem para o tratamento de feridas }\end{array}$ & $\begin{array}{l}\text { Encontros mensais com temas propostos pelo próprio grupo } \\
\text { de enfermagem. }\end{array}$ \\
\hline$B^{23}$ & $\begin{array}{l}\text { Cucolo,DF. } \\
\text { et al. }(2007)\end{array}$ & $\begin{array}{l}\text { Avaliação emancipatória de um programa educativo do } \\
\text { serviço de controle de infecção hospitalar }\end{array}$ & Entrevista coletiva realizado em quatro círculos de discussão. \\
\hline
\end{tabular}




\section{Quadro 2. Principais caracteristicas dos artigos revisados. Niterói, RJ, Brasil, 2019}

\section{Categorias}

País de publicação/
Continente

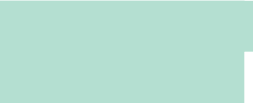

Periódico/foco e escopo

\section{Variantes}

América do Norte (EUA) ${ }^{13}$

Quantidade

$\begin{array}{cc}\text { América do Sul (Brasil) }{ }^{14,15,19,20,21,22,23,24} & 8 \\ \text { Continente Asiático (Índia) }{ }^{12} & 1 \\ \text { Europa (Itália) }{ }^{17} & 1 \\ \text { Oceania (Austrália) }{ }^{18} & 1\end{array}$

Journal of Hospital Infection; AJ Infect Control 24,25 2

Rev.Latino-Am Enfermagem; Cogitare Enferm; Acta Paul Enferm; Intensive and Critical Care Nursing; Rev.Eletr. Enf $13,14,15,20,21,23,24$

7

R.Pesq.Cuid.Fundam. On line ${ }^{19}$ Rev. Paul Pediatr 22 Journal of medical microbiology 12

Área de conhecimento do primeiro autor

Idioma de origem da publicação

Delineamento

Exploratória quantitativa ${ }^{12,17,18,19,21}$
Não controlada antes-depois ${ }^{14}$
Relato de experiência ${ }^{15,24}$

Coorte $^{22}$

Enfermagem ${ }^{13,14,15,19,20,21,23,24}$

Medicina $^{12,17,22}$

Infectologista ${ }^{18}$

Português $14,15,19,20,21,22,23,24$

Inglês ${ }^{12,13,17,18}$

Descritivo exploratória ${ }^{20}$

Qualitativa ${ }^{23}$

2

2
Houve uma redução significativa quanto ao risco associado à infecção ${ }^{12,17,22}$

Houve uma maior conscientização quanto ao conhecimento ao risco associado à infecção relacionada à saúde ${ }^{18} \quad 1$

Demonstrou uma melhoria na qualidade dos cuidados ${ }^{13}$

Foi de grande contribuição para o serviço de saúde ${ }^{19}$

tados

Favoreceu a construção de uma postura crítica e reflexiva, fez repensar nas práticas cotidianas favorecendo a transformação das atividades ${ }^{14,15,23,24}$

Permitiu o desenvolvimento das atividades fundamentadas no conhecimento de forma imediata e oportuna. ${ }^{20,21} \quad 2$

1A- Revisões sistemáticas e meta análises de ensaios clínicos comparáveis 12,13,17,18,19,21 6

2A- Revisão sistemática homogênea de estudos de coorte (com grupos de comparação e controle de variáveis) ${ }^{22} 1$

Níveis de evidência

Resposta à questão de pesquisa
2C- Resultados de pesquisas (observação de resultados terapêuticos ou evolução clínica) 14,20,23

4- Relatos de casos e série sem definição de caso controle ${ }^{15,24}$

Pôsteres, Cartazes e serviço de radiofonia $12,13,14,15$

Programa de informática ${ }^{24}$

Entrevista, reuniões e encontros mensais $17,20,21,22,23$

Capacitação e questionários ${ }^{18,19}$

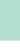

1


dância com as recomendações dessa política e que favoreçam o aprimoramento da educação em saúde.

Observou-se que $8,33 \%$ dos estudos na educação permanente foram direcionados aos profissionais do setor de limpeza e dos 91,7\% direcionados aos profissionais de saúde. A equipe de enfermagem participou da sua totalidade, $45,4 \%$ foram direcionados também a médicos, farmacêuticos, auxiliares de esterilização e atendentes e apenas 9\% direcionados a todos os profissionais do serviço de saúde, embora sendo estes os que potencialmente possuem importante influência na diminuição dos índices de morbidade e mortalidade dos pacientes e redução de custo, conforme um estudo que destaca a necessidade de que as ações estejam presentes no cotidiano de todos os profissionais de saúde, uma vez que não se faz prevenção sem envolvimento altruístico de todos.

A realização da educação permanente pode ser feita por todo profissional de saúde, pois o mesmo tem habilitação para execução, mas como o enfermeiro está em constante aquisição de conhecimento, habilidade e competência, justifica-se o seu maior envolvimento na EPS ${ }^{(11)}$.

Das estratégias empregadas, 33,3\% dos estudos utilizaram cartazes, folders e serviço de radiofonia; 41,7 reuniões, entrevista e encontros mensais; $16,7 \%$ capacitação e questionários e 8,33\% programa de informática. Dessa forma, para exibição das estratégias, foram organizadas categorias com seguintes subtítulos: comunicação audiovisual, metodologias ativas e programa de informática.

Meios auxiliares de aprimoramento do conhecimento quanto à prevenção e controle da infecção hospitalar

\section{Comunicação audiovisual}

Este tipo de estratégia está presente entre 04 artigos que mostraram a utilização cartazes que eram afixados em locais proeminentes e estratégicos. Ou- tro estudo apontou, além dos cartazes, a utilização de paródias musicais com a temática de higienização das mãos (HM) e frases construídas com palavras de intuito humorístico que, de certa forma, despertava inicialmente a curiosidade dos profissionais fazendo-os dar ênfase no conteúdo que estava sendo abordado levando-os à reflexão sobre o tema em questão, favorecendo a interação entre os profissionais na realização dos procedimentos de higiene das mãos ${ }^{(12-15)}$.

Na prevenção da Pneumonia Associada à Ventilação (PAV), foram utilizados cartazes storyboards que destacavam as melhores práticas existentes baseadas com o protocolo de cuidados orais, sendo desenhado para demarcar o papel do cuidado na prevenção da PAV. Foi publicado na Intranet do hospital para autoaprendizagem ${ }^{(13)}$.

Além da utilização dos cartazes, buscou-se conhecer o nível de entendimento dos profissionais sobre a HM por meio da aplicação do questionário, solicitando também que realizassem as etapas da HM e coletadas antes e depois da $\mathrm{HM}^{(12)}$.

Os resultados, segundo os autores(14), indicam que esse tipo de ferramenta foi eficaz visto que resultou na interação dos profissionais, possibilitou instigar os mesmos à reflexão crítica e, por conseguinte, maior adesão dos procedimentos favorecendo a prevenção e controle da infecção relacionada à saúde. Porém, há citações em que a utilização dessa estratégia por um período maior não foi significativa na adesão pelos profissionais, pois não levaram à reflexão crítica e, consequentemente, à mudança de comportamento.

Este tipo de estratégia corrobora com a proposta da Política de Educação Permanente em Saúde, que diz "a mudança das estratégias de organizações dos serviços e do exercício da atenção é construída na prática das equipes", ou seja, o profissional precisa refletir sobre sua realidade, os problemas existentes no seu cotidiano e, com isso, listar e priorizar as necessidades de capacitação e atualização ${ }^{(16)}$

O fato de a adesão pelos profissionais, através da utilização das ferramentas da educação permanente por um período maior não ter sido estatisticamente significativa e ter sido direcionada apenas aos profissionais que prestavam assistência diretamente aos RNs, supõe que no processo da educação, a ampliação do direcionamento para a equipe de saúde, inclusive a equipe de limpeza, poderia alcançar maior significância no período de considerado e, com isso, fazer diferença frente às ações realizadas por tais profissionais no controle da infecção hospitalar.

\section{Metodologias ativas}

Os estudos ${ }^{(17-23)}$ citam uma variedade de estratégias que foram conduzidas através de: reuniões mensais, ensino interativo na prática, capacitação, visitação diária do profissional do $\mathrm{CCIH}$ e entrevistas.

Durante os encontros mensais, os treinamentos e capacitação eram compostos por curta revisão de literatura, seguida de demonstração em vídeo acerca das práticas, levando-os à discussão sobre a atual prática com viabilidade para novas práticas. Em outro estudo ${ }^{(18)}$, incluiu-se também o fornecimento de folheto e visitação diária do profissional do $\mathrm{CCIH}$ no setor de enfermagem para esclarecimentos de dúvidas.

Além das estratégias utilizadas acima, estudo ${ }^{(19)}$ utilizou um questionário para avaliar o nível de conhecimento dos profissionais acerca da higiene hospitalar e mediante às respostas, foi desenvolvido um folder para ser entregue aos profissionais, no intuito de potencializar o conhecimento e orientar quanto às atividades diárias.

Observou-se em um artigo $^{(20)}$, a realização de entrevista individual para verificar o conhecimento das práticas educativas da equipe e com base nas respostas, possibilitou a reflexão crítica sobre possíveis modificações. 
Diferente do artigo acima, esse estudo realizou entrevistas coletivas, que levaram à discussão do processo educativo desenvolvido pelo Serviço de Controle de Infecção Hospitalar (SCIH), para verificar o processo e reestruturar com vistas a resultados positivos ${ }^{(30)}$.

Em dois $\operatorname{artigos}^{(21,22)}$, verificamos que utilizaram uma abordagem de intervenção educativa mais específica e pontual na qual a equipe se reunia mensalmente e utilizava os temas que eram propostos pela mesma, como o tema sobre as medidas de Prevenção e Infecção Corrente Sanguínea Relacionada a Cateter (ICSRC) e os cuidados com feridas. Empregou-se a metodologia de antes e depois, tendo sido observados os profissionais antes das intervenções e após, para verificar a variabilidade nas taxas de infecção bem como o índice de adesão proposta nas intervenções.

Diante do exposto, percebeu-se que foi empregada como estratégia, a metodologia ativa e, de acordo com os autores, as estratégias implementadas obtiveram maior adesão dos profissionais quanto aos procedimentos, despertando o senso crítico de suas práticas e possibilitando a transformação de suas atividades.

Essa estratégia enfatiza o uso de métodos criativos, dinâmicos e reflexivos, que são materializados na prática através dos seguintes procedimentos na educação: Problematização da realidade e do processo do trabalho, exercícios em grupo, leitura comentada, exposições dialogadas, debates temáticos, apresentação de filmes e interpretações musicais. A execução desta metodologia leva à reflexão crítica sobre as atividades diárias, possibilitando a transformação da sua realidade ${ }^{(16)}$.

Desse modo, o uso dessa metodologia está em conformidade com o que propõe a educação permanente, no uso de estratégias que viabilizem a integração de conhecimento de diversas áreas através de análise, avaliação e síntese que resulte na resolução de problemas e tomada de decisão ${ }^{(24)}$.
Acreditamos que esse tipo de estratégia possui um diferencial, pois estimula o envolvimento da equipe de saúde e permite a consolidação dos seus conhecimentos colocando-os na prática, estimulando o senso crítico e autonomia para possíveis propostas na mudança comportamental da prática.

\section{Programa de informática}

Nesse estudo, elaborou-se um programa educativo on-line e dinâmico contemplando suas funcionalidades num ambiente virtual de aprendizagem (AVA) com intuito de levar à reflexão crítica sobre as atividades diárias dos profissionais, possibilitando as interações que favoreçam a proposição de ideias e, assim, propicie a reconstrução dos processos de trabalho, proporcionando uma mudança da atual realidade ${ }^{(24)}$.

É importante ressaltar ainda uma característica importante na elaboração desse programa: os profissionais que idealizaram tiveram participação contínua como mediadores nesse processo, favorecendo as ações baseadas na reflexão crítica e permitindo que os profissionais elaborassem suas considerações finais e possibilidades, não interferindo no processo e favorecendo o espaço para críticas, reflexões e para propor novas intervenções. No entanto, essas intervenções aconteceram mediante discussão entre os profissionais da instituição que, em seu cotidiano, vivenciam a realidade do problema em questão ${ }^{(24)}$.

Acreditamos que o software utilizado é de grande valia, uma vez que alguns profissionais possuem limitações a aderirem ao processo da educação permanente presencial. Assim, essa ferramenta virtual, que flexibiliza a realização da educação, possibilita a inserção de todos os profissionais, proporcionando a interação com a equipe multidisciplinar, a troca de conhecimentos e experiências, levando-os à reflexão da prática atual e possíveis 
propostas de mudanças e medidas, que podem ser encaminhadas aos gestores que auxiliarão na busca de resoluções das dificuldades existentes na gestão e na prática profissional.

\section{CONCLUSÃO}

A educação permanente é uma proposta de intervenção fundamental na capacitação e qualificação dos profissionais, dando subsídios que levam à conscientização das necessidades reais de saúde e propiciam a mudança das práticas no âmbito do trabalho, favorecendo o atendimento de qualidade na assistência, possibilitando realizar com segurança os procedimentos e também o crescimento tanto do profissional quanto das organizações de saúde.

Nessa revisão, verificou-se que os estudos, em sua maioria, não aplicaram a educação permanente direcionada para equipe multidisciplinar, mas para profissionais da equipe médica e de enfermagem.

Na pesquisa, evidenciou-se, de forma clara, três tipos de estratégias e percebeu-se maior adesão nas metodologias ativas que colocavam os profissionais como agentes ativos no processo da educação e de construção de novas mudanças.

Ficou claro que a utilização de diversas estratégias da educação permanente na assistência à saúde traz melhorias à medida que estimulam os profissionais no aperfeiçoamento, qualificação e na atualização de conceitos e ações práti- cas, contribuindo também para o desenvolvimento da reflexão crítica, trazendo mudança comportamental, favorecendo, desta forma, o crescimento pessoal e profissional e a interação da equipe multidisciplinar propiciando a melhora na qualidade da assistência.

Portanto, é de suma importância a utilização da educação permanente na saúde, com foco na atualização da equipe multidisciplinar.

Dessa forma, frente à escassez de artigos e trabalhos publicados e, embora o assunto seja de alta relevância, percebeu-se, portanto, a necessidade do fomento de estudos voltados à educação permanente como forma de evidenciar a importância da prevenção e controle da infecção hospitalar.

\section{Referências}

1. Moura MEB, Campelo SMA, Brito FCP, Batista OMA, Araujo TME, Oliveira ADS. Infecção hospitalar: estudo de prevalência em um hospital público de ensino. Rev Bras enferm. Brasília. 2007; 60(4):416-21.

2. Leiser JJ, Tognim MCB, Bedendo J. Infecções Hospitalares em um Centro de Terapia Intensiva de um hospital de ensino do Norte do Paraná. Cienc Cuid Saude. 2007 Abr/Jun; 6(2):181-186.

3. Perna TDGS, Puiatti MA, Pereira NMM, Couri MG, Ferreira CMD. Prevalência de infecção hospitalar pela bactéria do gênero klebsiella em uma Unidade de Terapia Intensiva. Rev Soc Bras Clin Med.UFJF. 2015 abr-jun; 13(2):119-23.

4. Organização Mundial da Saúde. Prevenção e controle de infecção. Infecções associadas aos cuidados de saúde. OMS, 2018.

5. Organização Mundial da Saúde. Relatório da OMS sobre o ônus das infecções associadas aos cuidados de saúde endêmicos em todo o mundo: Uma revisão sistemática da literatura. OMS, 2011.

6 . World Health Organization. Delivering quality health services: a global imperative for universal health coverage. WHO, 2018.

7. Agência Nacional de Vigilância Sanitária (BR). Programa Nacional de Prevenção e Controle de Infecções Relacionadas à Assistência à Saúde (2013 - 2015). ANVISA, 2013.

8. Pereira SB, Backes DS. Martins FDP, Rangel RF. Educação Permanente em uma unidade obstétrica na perspectiva de profissionais de saúde. Rev. enferm UFPE. 2017; 11 (supl.3):1458-63.

9. Massaroli A, Saupe R. Distinção Conceitual: Educação Permanente e Educaç邓o Continuada no processo de trabalho em saúde. PIBIC, 2008.

10. Pedrosa KK, Oliveira ICM, Feijão AR, Machado RC. Enfermagem baseada em evidência: caracterização dos estudos no Brasil. Cogitare Enferm. 2015; 20(4):733-74.

11. Santana AS, Rodrigues, CSS, Bantim IO, Souza LM, Portela PP, Silva TS. Influência da educação permanente em saúde para redução das taxas de infecção na Unidade de terapia Intensiva. COVINBRA, 2017.

12. Kapil R, Bhavsar HK, Madan M. Hand Hygiene in reducing trasient flora on the hands of health care workers:an education intervention. Indian Journ of Med Microb. 2015; 33(1):125-28.

13. Ross A, Crumpler J. The impact of an evidence-based practice education on the role of oral care in the prevention of ventilator-associated pneumonia. Elsevier. 2007; 23:132-36.
14. Neves ZCP, Tipple AFV, Souza ACS, Pereira MS, Melo DS, Ferreira LR. Higienização das mãos: o impacto de estratégias de incentivo à adesão entre profissionais de saúde de uma Unidade de terapia Intensiva Neonatal. Rev. Latino-Am. 2006; 14(4).

15. Neves ZCP, Tipple AFV, Souza ACS, Ferreira LR, Silva EAC. Relato de experiência: utilização de cartazes estilizados como medida de incentivo à higienização das mãos. Rev. Eletr.Enf. 2009; 11(3):738-45.

16. Cury MC, Mello KS. Metodologias ativas na educação permanente em saúde: Uma revisão de literatura . UNC. Curso de pós-graduação lato sensu em gestão de saúde. Curitibanos, 2016.

17. Musu M,Finco G, Mura P, Landoni G, Piazza MF, Messina M, Tidore M, et al. Controlling Catheter-related bloods tream infections trough a multicenter education program for intensive care units. Journ of Hosp Infect. 2017; 97(3):275-81.

18. Gillespie E, Rodrigues A, Wright L, Williams N, Stuart RL. Improving antibiotic stewardship by involving nurses. American Journ of Infec Cont. 2013; 41(4):365.

19. Valente GSC, Falcão PM, Barbosa SQ, Rosa SGM, Santos WA, Barbosa VQ. 0 enfermeiro na educação em saúde do trabalhador da limpeza no ambiente hospitalar. R. pesq.:cuid fundam. 2011; 3(1):1702-10.

20. Souza MCB, Ceribelli MIP. Enfermagem no centro de material esterilizado- a prática da educação continuada. Rev.Latino-Am Enfermagem. 2004; 12(5):767-74.

21. Jacondino CB, Severo DF, Rodrigues KR, Lima L, Einhardt RR, Amestoy SC.Educação em serviço: qualificação da equipe de enfermagem para o tratamento de feridas. Cogitare Enferm. 2010; 15(2):314-8.

22. Vilela R, Dantas SRPE, Trabasso P. Equipe Multidisciplinar reduz infecção sanguínea relacionada ao cateter venoso central em Unidade de Terapia Intensiva Pediátrica. Rev. Paul.Pediatr. 2008; 28(4):292-98.

23. Cucolo DF, Faria JIL, Cesariano CB. Avaliação emancipatória de um programa educativo do serviço de controle de infecção hospitalar. Acta Paul Enferm. 2007; 20(1):49-54.

24. Silva LMG, Gutiérrez MGR, Domenico EBL. Relato de experiência: Ambiente virtual de aprendizagem na educação continuada em enfermagem. Acta Paul Enferm. 2010; 23(5):70-4. 